\title{
SPARC inhibits breast cancer bone metastasis and may be a clinical therapeutic target
}

\author{
JINGJING MA ${ }^{1}$, SHENG GAO $^{1}$, XIJU XIE $^{2}$, ERHU SUN $^{1}$, MIN ZHANG $^{1}$, QIAN ZHOU $^{1}$ and CHENG LU $^{1}$ \\ ${ }^{1}$ State Key Laboratory of Reproductive Medicine, Department of Breast Surgery, Nanjing Maternity and \\ Child Health Care Hospital Affiliated to Nanjing Medical University; ${ }^{2}$ Department of Breast Surgery, \\ The First Affiliated Hospital of Nanjing Medical University, Nanjing, Jiangsu 210029, P.R. China
}

Received July 9, 2015; Accepted February 13, 2017

DOI: $10.3892 / \mathrm{ol} .2017 .6925$

\begin{abstract}
Breast cancer is one of the most common types of cancer in females worldwide, and metastasis to bone is an important characteristic of malignancy. The present study aimed to investigate the molecular mechanism of breast cancer to bone metastasis of secreted protein acidic and rich in cysteine (SPARC). Immunohistochemistry was performed to examine the expression of SPARC in primary breast tumors and bone metastatic foci. Western blotting and reverse transcription-quantitative polymerase chain reaction were performed to detect the expression level of SPARC in several types of breast cancer cell. A Transwell filter assay was used to assess the effect of SPARC on breast cancer cell invasion ability, and an osteoblast differentiation assay was employed to analyze the effect of SPARC on the differentiation ability of mesenchymal stem cells. Clinical data revealed that decreased stromal SPARC expression is associated with breast cancer to bone metastasis. Gain- and loss-of-function studies reveal that SPARC inhibits the migration and invasion of breast cancer cells, and suppresses osteoclast activation in the breast cancer microenvironment. SPARC serves an important role in breast cancer bone metastasis and may be a promising therapeutic target for the treatment of breast cancer bone metastasis.
\end{abstract}

\section{Introduction}

Breast cancer is a common malignant tumor in females and the second most common cause of cancer-associated mortality (1). In total, 60 to $70 \%$ of patients with metastatic breast cancer exhibit bone metastases (2). Therefore, identifying the

Correspondence to: Professor Cheng Lu, State Key Laboratory of Reproductive Medicine, Department of Breast Surgery, Nanjing Maternity and Child Health Care Hospital Affiliated to Nanjing Medical University, 123 Tianfei Lane, Gulou, Nanjing, Jiangsu 210029, P.R. China

E-mail: luchengshuaige@163.com

Key words: breast cancer, bone metastasis, secreted protein acidic and rich in cysteine mechanisms of tumor metastasis to bone is critical to therapeutic approach.

Secreted protein acidic and rich in cysteine (SPARC), also termed osteonectin or basement-membrane protein 40 , is a 34-kDa, calcium-binding glycoprotein that associates with cell membranes and membrane receptors (3). Bellahcene and Castronovo (4) suggested that increased expression of SPARC in malignant breast tumors may serve a role in the preferential homing of breast cancer cells to bones. Osteonectin is a factor in bone extracts that promotes breast and prostate cancer cell invasion to bone in vitro (5). Additionally, bone extracts from osteonectin-null mice exhibit reduced chemoattractant activity for prostate cancer cells (6).

Several studies have revealed that increased SPARC expression is associated with poor prognosis with respect to breast cancer: Helleman et al (7) reported that SPARC expression levels were significantly associated with a shorter metastasis free survival; Hsiao et al (8) reported that patients with positive SPARC expression had 2.34 times higher risk of mortality compared with those with negative SPARC expression level following adjusting for factors including positive lymph node, tumor-node-metastasis tumor stage, estrogen receptor and progesterone receptor; however, Koblinski et al (9) reported the opposite result. Koblinski et al (9) reported that increased endogenous expression of SPARC may inhibit the invasive activity of breast cancer cells and reduce tumor cell-platelet aggregation.

To investigate the function of SPARC in breast cancer bone metastasis, the present study evaluated the predictive value of SPARC in primary breast cancer and bone metastatic foci. The effect of endogenous expression of SPARC on the invasion ability of breast cancer cells and bone metastasis was then determined. The present study revealed that increased SPARC expression correlated with a low rate of bone metastasis, and SPARC may inhibit migration and invasion in vitro. SPARC may also suppress osteoclast activation in the breast cancer microenvironment. These results suggest that SPARC serves an important role in breast cancer bone metastasis and may be a promising therapeutic target for the treatment of breast cancer bone metastasis.

\section{Materials and methods}

Human tumor cell lines and cell culture. The breast cancer MDA-MB-231, BT474, MCF-7, SKBR3, MCF10A, 
HCC1937, T47D and ZR-75-30 cell lines were purchased from the American Type Culture Collection (Manassas, VA, USA) and cultured in Dulbecco's modified Eagle's medium (DMEM; Gibco; Thermo Fisher Scientific, Inc., Waltham, MA, USA). The SUM1315 cell line was provided by Dr Stephen Ethier (University of Michigan, Ann Arbor, MI, USA). SUM1315-bo cells were established and termed as in our previous study (10). SUM1315-bo cells are derived from SUM1315 metastatic tumor in implanted bone. All cells were maintained at $37^{\circ} \mathrm{C}$ in a humidified chamber supplemented with $5 \% \mathrm{CO}_{2}$.

Patient selection. Formalin-fixed paraffin-embedded tumor samples $(n=50)$ were collected at the Department of Breast Surgery, Nanjing Maternity and Child Health Care Hospital Affiliated to Nanjing Medical University (Nanjing, China) and the Department of Breast Surgery, The First Affiliated Hospital of Nanjing Medical University (Nanjing, China) between February 2002 and March 2007 from patients who were surgically treated for clinical stage I-III breast cancer (aged 34-65 years) (11). The patients of the present study were followed up until December 2013 to determine whether they were positive or negative for bone metastasis, with a median follow-up time of 64 months (range, 50-78 months). Patient characteristics are listed in Table I. All patients provided informed consent, and the present study was approved by the Ethical and Scientific Committee of Nanjing Maternity and Child Health Care Hospital Affiliated to Nanjing Medical University (Nanjing, China).

Antibodies and reagents. SPARC antibody (\#5420) was purchased from Cell Signaling Technology, Inc. (Danvers, MA, USA). Goat anti-rabbit immunoglobulin G ( $\mathrm{IgG}$ )-horseradish peroxidase secondary antibodies (BS10003) and antibodies against GAPDH (MB001) were purchased from Bioworld Technology, Inc. (St. Louis Park, MN, USA).

Plasmids and viral production. Wild-type SPARC open reading frame (ORF) was isolated from human SUM1315 breast cancer cells complementary DNA using polymerase chain reaction (PCR). The primers used were as follows: Forward, 5'-GGAAGAAACTGTGGCAGAGG-3' and reverse, 5'-ATTGCTGCACACCTTCTCAA-3'. The ORF was 1983 bp in length. The fragment was cloned into pGFP-LV5 via a NotI/NsiI site. The recombinant lentiviral pGFP-LV5-SPARC expression plasmid was packaged into a mature lentivirus using 293T cells (American Type Culture Collection), and the supernatant $\left(4^{\circ} \mathrm{C}, 1,000 \mathrm{x} \mathrm{g}, 15 \mathrm{~min}\right)$ containing the virus was harvested, concentrated and titrated. Transfection with pGFP-LV5 empty vector was used as a control. SUM1315 cells were subsequently infected using the recombinant lentiviral vector. Flow cytometry was used to select the green fluorescent protein-cells. Western blot analysis was performed to detect the SPARC expression level (10).

Transfection. The oligonucleotides were transfected into SUM1315 and SUM1315-bo cells with Lipofectamine 2000 reagent (Invitrogen; Thermo Fisher Scientific, Inc.) at $80-90 \%$ confluence according to the manufacturer's protocol. All oligonucleotides, small interfering RNAs (siRNAs)
Table I. Association between stromal SPARC expression and the clinicopathological characteristics of patients with breast cancer.

\begin{tabular}{|c|c|c|c|}
\hline Characteristic & $\begin{array}{l}\text { Patients, } \\
\mathrm{n}\end{array}$ & $\begin{array}{l}\text { Patients with } \\
\text { high SPARC } \\
\text { expression, } \\
\text { n }(\%)\end{array}$ & P-value \\
\hline Age, years & & & 0.095 \\
\hline$\leq 50$ & 28 & $9(32.1)$ & \\
\hline$>50$ & 22 & $2(9.0)$ & \\
\hline Bone metastasis & & & 0.001 \\
\hline Yes & 25 & $2(8.0)$ & \\
\hline No & 25 & $13(52.0)$ & \\
\hline Tumor size ${ }^{a}$ & & & 0.454 \\
\hline $\mathrm{T} 1$ & 20 & $4(20.0)$ & \\
\hline $\mathrm{T} 2$ & 22 & $4(18.1)$ & \\
\hline $\mathrm{T} 3$ & 8 & $3(37.5)$ & \\
\hline Node stage $^{a}$ & & & 0.087 \\
\hline No & 9 & $1(11.1)$ & \\
\hline N1 & 23 & $3(13.0)$ & \\
\hline $\mathrm{N} 2$ & 14 & $6(42.8)$ & \\
\hline N3 & 4 & $1(25.0)$ & \\
\hline Histological grade & & & 0.708 \\
\hline 1 & 2 & $0(0.0)$ & \\
\hline 2 & 39 & $9(23.0)$ & \\
\hline 3 & 9 & $2(22.2)$ & \\
\hline ER & & & 0.728 \\
\hline Positive & 17 & $3(17.6)$ & \\
\hline Negative & 33 & $8(24.2)$ & \\
\hline PR & & & 1.000 \\
\hline Positive & 14 & $3(21.4)$ & \\
\hline Negative & 36 & $8(22.2)$ & \\
\hline HER $2 ~^{\text {b }}$ & & & 0.651 \\
\hline Positive & 10 & $4(30.0)$ & \\
\hline Negative & 40 & $7(20.0)$ & \\
\hline
\end{tabular}

${ }^{\text {aT }}$ Tumor-Node-Metastasis classification according to the International Union for Cancer Control criteria. ${ }^{b} \mathrm{HER} 2$ positivity, assessed as positive fluorescent in situ hybridization test. Significance was assessed using a $2 \times 2$ table and the $\chi^{2}$ test. ER, estrogen receptor; PR, progesterone receptor; SPARC, secreted protein acidic and rich in cysteine.

of SPARC, GGAAGAAACUGUGGCAGAGGUGACU (si-S1); CAAGAACGUCCUGGUCACCCUGUA U (si-S2); GCGGGUGAAGAAGAUCCAUGAGAAU (si-S3), were obtained from Invitrogen (Thermo Fisher Scientific, Inc.). The oligonucleotides were chemically modified (20-O-Methyl) oligos. They were revealed to exert long-term effects ( 2 weeks) and high gene knockout efficiency. Transfection efficiency was analyzed using a fluorescence microscope $24 \mathrm{~h}$ subsequent to transfection and defined as the number of cells capable of exhibiting fluorescence divided by the number of untransfected controls. 
Reverse transcription-quantitative (RT-q)PCR analysis. Total RNA was extracted using TRIzol total RNA isolation reagent (Invitrogen; Thermo Fisher Scientific, Inc.) according to the manufacturer's protocol. Specific primers from Invitrogen; Thermo Fisher Scientific, Inc., (Shanghai, China) were used for transcript detection: Actin forward, 5'-CTCCATCCT GGCCTCGCTTGT-3' and reverse, 5'-GCTGTCACCTTC ACCGTTCC-3'; SPARC forward, 5'-GGAAGAAACTGT GGCAGAGG-3' and reverse, 5'-ATTGCTGCACACCTT CTCAA-3'. qPCR (5X PrimeScript buffer $2 \mu$, PrimeScript RT Enzyme Mix $10.5 \mu \mathrm{l}$, Oligo Dt Prime $0.5 \mu \mathrm{l}$, Random 6 mers $0.5 \mu \mathrm{l}$, total RNA and RNase Free $\mathrm{dH}_{2} \mathrm{O}$ up to $10 \mu \mathrm{l}$; Takara Bio, Inc., Otsu, Japan) was performed with SYBR Green I (Takara Bio, Inc.). The progression consisted of 40 cycles $\left(95^{\circ} \mathrm{C}\right.$ for $15 \mathrm{sec}$ and $60^{\circ} \mathrm{C}$ for $\left.1 \mathrm{~min}\right)$ subsequent to an initial denaturation step $\left(95^{\circ} \mathrm{C}\right.$ for $\left.10 \mathrm{~min}\right)$. The mean number of three independent analyses for each gene and sample was calculated and normalized to the endogenous GAPDH (12) reference control gene actin using the $2^{-\Delta \Delta \mathrm{Cq}}$ method (13).

Western blot analysis. The cells were harvested in radioimmunoprecipitation assay lysis buffer (Beyotime Institute of Biotechnology, Shanghai, China). Samples were incubated for $1 \mathrm{~h}$ on ice with agitation and centrifuged at $4^{\circ} \mathrm{C} 12,000 \mathrm{x} \mathrm{g}$ for $20 \mathrm{~min}$. A total of $10 \mathrm{~g}$ protein samples (based on concentration) were subject to electrophoresis on $12 \%$ SDS-PAGE, transferred to a polyvinylidene difluoride membrane, blocked in 5\% nonfat milk in TBS-Tween-20 and hybridized with antibodies against SPARC (dilution, 1:1,000) and GAPDH (dilution, 1:5,000). GAPDH was used as a loading control. Signals were determined subsequent to incubation with horseradish peroxidase-conjugated anti-rabbit IgG secondary antibody (dilution, 1:1,000) using enhanced chemiluminescence. Protein expression levels were evaluated by densitometric analysis (Quantity One software version 4.62; Bio-Rad Laboratories, Inc., Hercules, CA, USA).

Transwell migration and invasion assays. The in vitro invasion studies were performed using a BD BioCoat Matrigel invasion assay system (BD Biosciences, Franklin Lakes, NJ, USA). Cells were seeded at $5 \times 10^{4} /$ well on $8-\mu$ m pore Transwell inserts (Corning Inc., Corning, NY, USA). The lower chamber was filled with DMEM containing $10 \%$ fetal bovine serum (Gibco; Thermo Fisher Scientific, Inc.). Subsequent to incubation for $24 \mathrm{~h}$, the number of cells in the lower chamber of the filter membranes was determined. All in vitro experiments were performed in triplicate, and all trials produced similar results. For Transwell migration assays, the cells were plated at $1 \times 10^{5} /$ well in the upper chamber on an $8-\mu \mathrm{m}$ membrane (BD Biosciences) precoated with $100 \mu \mathrm{g} / \mathrm{ml}$ fibronectin and $2.5 \%$ bovine serum albumin (both from Gibco; Thermo Fisher Scientific, Inc.). Cells were incubated for the indicated lengths of time $(8 \mathrm{~h})$ under standard culture conditions. Tumor cells remaining on the upper surface of the membrane were removed and cells that had migrated to the underside were fixed and stained with $0.1 \%$ crystal violet for $30 \mathrm{~min}$, rinsed in PBS and subjected to microscopic inspection (Omega Bio-Tek, Inc., Norcross, GA, USA). A total of five images of the preset fields per insert were captured. Subsequent to staining and the capturing of images, the cells that had migrated to the underside were eluted using $33 \%$ acetic acid, and the optical density values of absorbance in the positively stained cells were measured using a microplate reader (Omega Bio-Tek, Inc.) at $570 \mathrm{~nm}$.

Preparation of conditioned medium (CM). The SUM1315 cells were grown until sub-confluence, and subsequently starved in serum-free DMEM for $24 \mathrm{~h}$. The CM was collected, centrifuged $\left(4^{\circ} \mathrm{C}, 1,000 \mathrm{x} \mathrm{g}, 15 \mathrm{~min}\right)$, concentrated and aliquoted, and stored at $-20^{\circ} \mathrm{C}$ until use.

Osteoblast differentiation and osteoclastogenesis assay in vitro. Human mesenchymal stem cells (HMSCs) were purchased from ScienCell (Carlsbad, CA, USA), plated in cell culture flasks and expanded in mesenchymal stem cell growth medium (MSCM; ScienCell) at $37^{\circ} \mathrm{C}$ in a $5 \% \mathrm{CO}_{2}$ atmosphere for 7-10 days. Subsequent to having grown to an adequate density (70-80\% convergence), the adherent cells were trypsinized and seeded at a density of 5,000 cells $/ \mathrm{cm}^{2}$ on a 24-well plastic plate. After $24 \mathrm{~h}, \mathrm{MSCM}$ was removed, and osteogenic induction medium (ScienCell) was added to induce osteoblast differentiation (day 0). On the seventh day, the osteoblast cells were plated at $1 \times 10^{6}$ cells per well in 24 -well plates, at a 1:1 ratio of basal culture medium/filtered CM (harvested from $24 \mathrm{~h}$ incubation of confluent tumor cells). The medium was replaced every 2 days. Tartrate-resistant acid phosphatase (TRAP) staining was performed on day 7 using a Leukocyte Acid Phosphatase kit from Sigma-Aldrich; Merck KGaA (Darmstadt, Germany) (14). TRAP-positive multinucleated cells were considered mature osteoclasts and were included in the number of osteoclasts per well.

Immunohistochemistry (IHC). Paraffin-embedded tissue sections were deparaffinized, rehydrated, rinsed, immersed in $10 \mathrm{mM}$ sodium citrate, microwaved for $20 \mathrm{~min}$ and cooled for $20 \mathrm{~min}$. For immunocytohistochemistry, the slides were fixed in PBS ( $\mathrm{pH}$ 7.4) solution containing 4\% paraformaldehyde for $10 \mathrm{~min}$ and rinsed. Subsequent to incubation in methanol containing $3 \%$ hydrogen peroxide for $10 \mathrm{~min}$ to block endogenous peroxidase activity, the slides were microwaved in $0.01 \mathrm{mmol} / 1$ sodium citrate $(\mathrm{pH} \mathrm{6.0)}$ for antigen retrieval, incubated with rabbit polyclonal antibody against SPARC (1:1,000; Cell Signaling Technology, Inc.) for $2 \mathrm{~h}$ at $37^{\circ} \mathrm{C}$, incubated with horseradish peroxidase-labeled rabbit anti-goat secondary antibody for $1 \mathrm{~h}$ at $37^{\circ} \mathrm{C}$, incubated with 3,3'-diaminobenzidine solution for $10 \mathrm{~min}$ and counterstained with hematoxylin.

Evaluation of IHC results. Immunohistochemical staining results were interpreted by two experienced pathologists, and the mean staining density was determined using Image-Pro Plus 6.0 (Media Cybernetics, Inc., Rockville, MD, USA). SPARC expression was evaluated under a light microscope at magnification, $x 400$. For each specimen, five images of representative areas were acquired, and a total of 1,000 to 2,000 tumor cells were counted. For human samples, IHC scoring was performed using a modified histoscore, which included a semi-quantitative assessment of the fraction of positive cells and the intensity of staining. The extent of the staining, defined as the relative area of positive staining within the tumor cells 


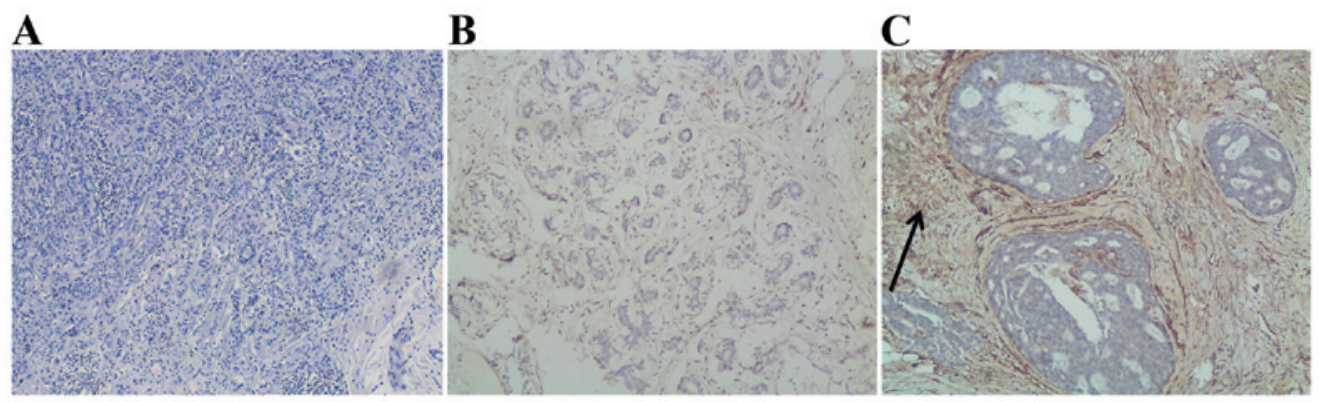

Figure 1. Representative images of SPARC immunohistochemical staining in breast cancer bone metastasis patients. (A) Negative control breast cancer sample. Magnification, x100. (B) Weak expression of SPARC in breast cancer sample. Magnification, x100. (C) Strong staining of SPARC in breast cancer sample. Magnification, $x 100$. Arrow indicates SPARC positive staining. SPARC, secreted protein acidic and rich in cysteine.

relative to the entire tissue area, was scored on a scale of $0-4$ as follows: $0,0-10 \% ; 1,11-25 \% ; 2,26-50 \% ; 3,51-75 \%$; and 4 , $>75 \%$. The sum of the staining-intensity and staining-extent scores was used as the final staining score for SPARC (0-7). For the statistical analysis, a final staining score of 0-5 was considered indicative of low expression, and scores of 6-7 were considered indicative of high expression. The immunostained slides were evaluated by two board-certified pathologists at two separate institutions (Nanjing Maternity and Child Health Care Hospital Affiliated to Nanjing Medical University and The First Affiliated Hospital of Nanjing Medical University, Nanjing, China). The pathologists independently examined the entire tissue section and were blinded to the clinical data. Reading agreement was found to be $96 \%$ concordant between the two pathologists. Non-concordant cases were resolved by a third pathologist who blindly scored those cases, and the two out of three rule was used for the determination of final scores.

Statistical analyses. The data are presented as the mean \pm standard deviation. A Student's t-test (two-tailed) was used to determine the statistical significance of the differences between the groups. $\mathrm{P}<0.05$ was considered to indicate a statistically significant difference. The intensity of SPARC expression in human breast cancer samples was analyzed using the $\chi^{2}$ test. Statistical analysis was performed using SPSS version 19.0 software (IBM SPSS, Armonk, NY, USA).

\section{Results}

Association between stromal SPARC expression and breast cancer bone metastasis. Stromal SPARC expression was evaluated in 50 patients with breast cancer using IHC. Representative images of SPARC staining are shown in Fig. 1. High stromal SPARC expression was associated with a decreased risk of bone metastasis in patients with breast cancer. Myoepithelial cells exhibited significantly strong stromal SPARC expression in $8 \%(2 / 25)$ and $52 \%(13 / 25)$ of the patients, respectively (Table I). The association between stromal SPARC expression and breast cancer bone metastasis was analyzed. High levels of stromal SPARC expression were closely associated with decreased levels of breast to bone metastasis $(\mathrm{P}=0.001)$.

SPARC expression in a panel of breast cancer cells. The SUM1315-bo and SUM1315 cells exhibited increased
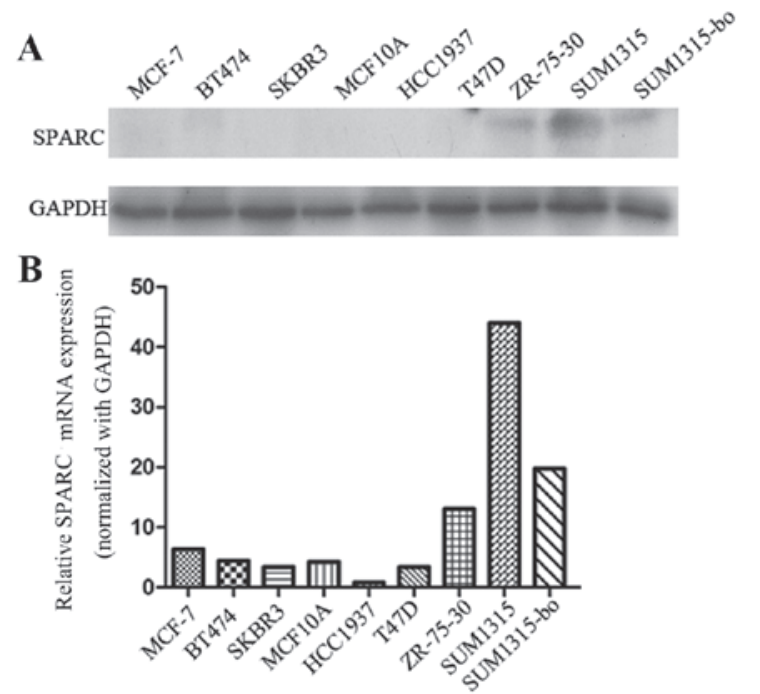

Figure 2. SPARC expression in a panel of breast cancer cells. (A) Protein and (B) mRNA levels of SPARC expression were assessed in a panel of breast cancer cell lines. SPARC expression was markedly increased in SUM1315 and SUM1315-bo cells compared with the other breast cancer cells. SPARC, secreted protein acidic and rich in cysteine.

SPARC protein levels compared with the MCF-7, BT474, SKBR3, MCF10A, T47D, ZR-75-30 and HCC1937 cells. The present study revealed that SUM1315-bo cells exhibited reduced SPARC expression compared with SUM1315 cells (Fig. 2).

Inhibition of endogenous SPARC and migration and invasion of SUM1315 cells. The role of SPARC in SUM1315 cell migration and invasion was investigated by knocking down SPARC using siRNA. The present study used SUM1315 cells as they exhibit a high migratory potential and express endogenous SPARC at high levels. A total of three siRNA-coding oligos against human SPARC were designed and compared. The most effective SPARC siRNA construct exhibited a target sequence of GCGGGUGAAGAAGAUCCAUGAGAAU (si-S3). This sequence was verified at the mRNA and protein levels (Fig. 3A). Notably, SPARC silencing was associated with significantly increased invasion and migration in SUM1315 cells (Fig. 3B).

Overexpression of SPARC and invasion and migration of SUM1315-bo cells. The present study transfected 
A

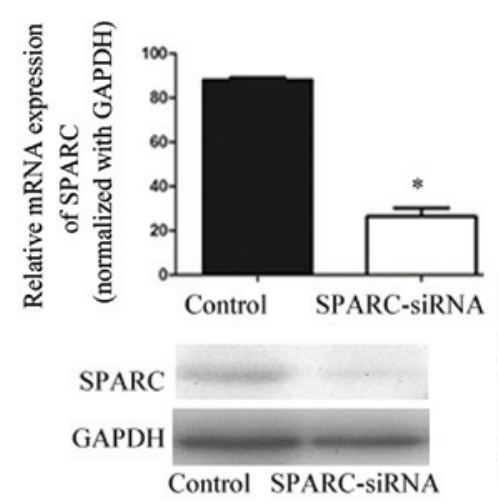

C

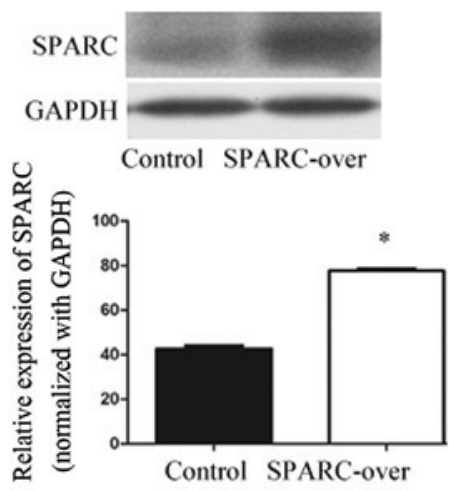

B

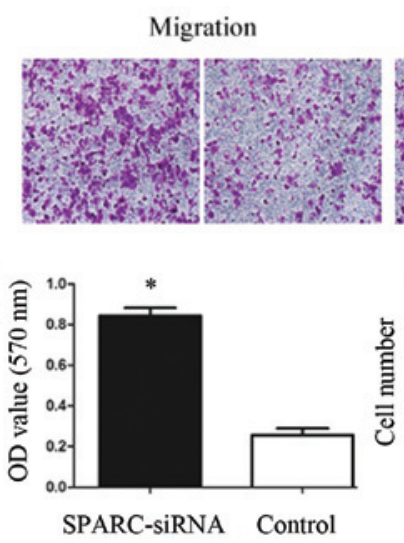

D
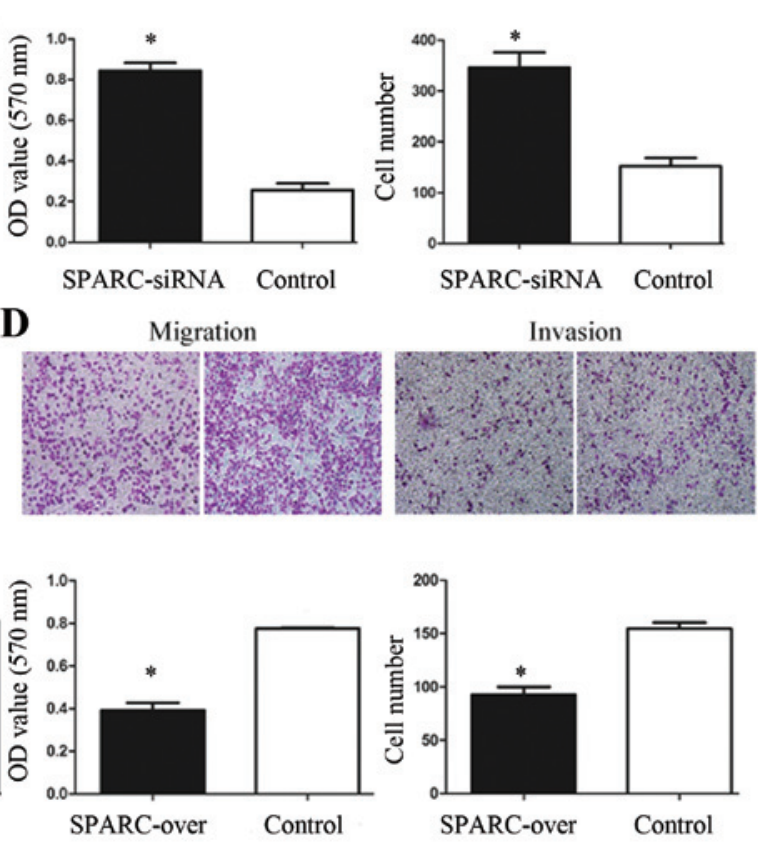

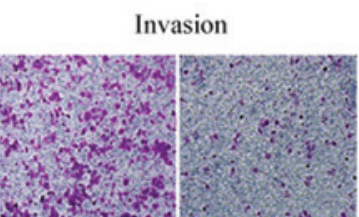

Invasion

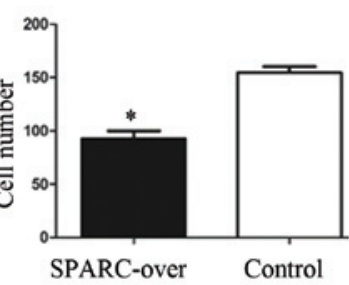

Figure 3. Effect of SPARC on migration and invasion of breast cancer cells. (A) siRNA of SPARC was transfected into SUM1315, which was confirmed at the mRNA and protein levels. (B) Migration and Invasiveness of SPARC knockdown SUM1315 and control cells was assessed using Transwell assays. The invasiveness through $8-\mu \mathrm{m}$ pore Transwell inserts was significantly increased in SPARC knockdown SUM1315 compared with the negative control. Magnification, x100. (C) Constructed expression vector pEGFP-C2-SPARC was stably transfected into SUM1315-bo. Transfection was confirmed at the mRNA and protein levels. (D) Migration and Invasiveness of SPARC-over and control cells were assessed by Transwell assays. The invasiveness through 8- $\mu \mathrm{m}$ pore Transwell inserts was revealed to be significantly lower in SPARC-over compared with the controls. Magnification, x100. ${ }^{*}<0.05$. SPARC, secreted protein acidic and rich in cysteine; siRNA, small interfering RNA; control, negative control; SPARC-over, SPARC overexpressing SUM1315-bo; OD, optical density.

$\mathbf{A}$

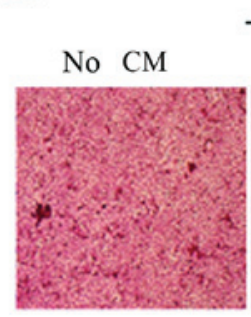

With conditioned media

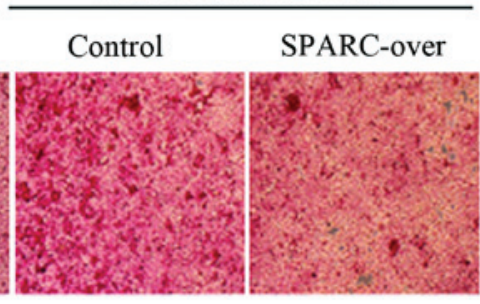

B

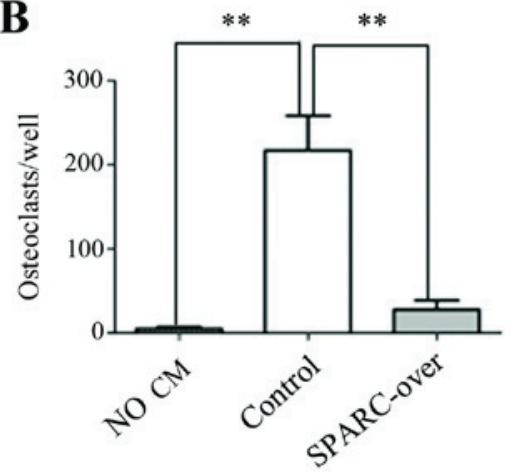

Figure 4. SPARC inhibits osteoclastogenesis in tumor cells. Induction of osteoclastogenesis in human mesenchymal stem cell-derived osteoblasts by CM from SUM1315-bo cells. Differentiated multinucleated osteoclasts were revealed by TRAP staining. (A) Representative images of TRAP-staining. (B) Quantification of TRAP-positive cells. Data are presented as the mean \pm standard deviation. ${ }^{* *} \mathrm{P}<0.01$ with two-sided Student's t-test. CM, conditioned medium; TRAP, tartrate-resistant acid phosphatase; SPARC, secreted protein acidic and rich in cysteine; SPARC-over, SPARC overexpressing SUM1315-bo.

SUM1315-bo cells with the constructed expression vector pGFP-LV5-SPARC to determine whether SPARC overexpression decreased the level of cell migration and invasion. Using western blot analysis, it was revealed that SPARC levels increased 2.12-fold subsequent to transfection (Fig. 3C). The effects of SPARC on the migratory and invasive behavior of
SUM1315-bo cells were analyzed. The results identified a 2.13 -fold decrease in cell motility and a 2.32-fold decrease in cell invasiveness subsequent to transfection of the constructed expression vector pGFP-LV5-SPARC (Fig. 3D). These results indicated that SPARC overexpression inhibited migration and invasion of SUM1315-bo cells in vitro. 
SPARC inhibits tumor-stimulated osteoclast activation in SUM1315-bo cancer cells. The present study investigated the role of SPARC in tumor-stimulated osteoclast activation. HMSC-derived osteoblasts were treated with CM from the control and SPARC-overexpression sublines of SUM1315-bo cells in an in vitro osteoclastogenesis assay, and TRAP-positive multinucleated mature osteoclasts were scored (Fig. 4). HMSC-derived osteoblasts were cultured with $\mathrm{CM}$ from tumor cells and exhibited numerous TRAP-positive cells. The CM of the SUM1315-bo cell line induced extensive osteoclast differentiation. However, the osteoclast-activating ability of the CM was significantly decreased when SPARC expression was overexpressed.

\section{Discussion}

The present study aimed to elucidate the function of SPARC in breast cancer bone metastasis. The overexpression of SPARC stroma expression was revealed to be associated with a decreased risk of bone metastasis in patients with breast cancer.

SPARC, also termed osteonectin, is a $32-\mathrm{kDa}$ secreted glycoprotein that interacts with extracellular matrix (ECM) proteins to promote the disassociation of cells from the matrix, thereby promoting cell motility. SPARC serves an important role in wound healing, embryonic development and tumorigenesis (15). In addition to interacting with ECM components, SPARC interacts with growth factors, including vascular endothelial growth factor and fibroblast growth factor.

Increased SPARC expression has been identified in multiple types of tumor and is associated with poor prognosis $(12,16,17)$. In breast carcinoma, SPARC has been identified as a member of a cluster of genes associated with increased invasive capacity (18). In addition, the mRNA levels of SPARC inversely correlate with estrogen receptor status, indicating that SPARC expression is associated with more aggressive types of breast cancer (12). The role of SPARC in tumorigenesis is complex as it is expressed in epithelial and stromal compartments. Notably, epithelial SPARC expression does not confer a poorer prognosis in lung and pancreatic cancer, whereas stromal SPARC expression has been associated with poor clinical outcomes independent of common clinicopathological parameters $(16,19)$. The mechanism by which stromal SPARC expression confers a poor prognosis is not known. A potential mechanism by which SPARC promotes tumorigenesis is through the stimulation of angiogenesis. SPARC was initially identified as a protein secreted by endothelial cells in vitro (20). Increased SPARC expression was detected in newly formed vessels in malignant melanoma xenografts and during neovascularization of aortic stenosis $(21,22)$. SPARC has also been demonstrated to mediate several stages of the epithelial-to-mesenchymal transition, and its expression is a feature of metaplastic breast carcinoma (23).

Both gain- and loss-of-function studies have revealed that SPARC inhibits breast cancer cell migration and invasion. Furthermore, SPARC inhibits the osteoclast-activating ability of the CM in SUM1315 cancer cells. Therefore, chemotherapy agents targeting SPARC may be suitable to reverse the potential of breast cancer cells for bone metastasis.

\section{Acknowledgements}

The present study was supported by the National Natural Science Foundation of China (grant no. 81172501).

\section{References}

1. DeSantis C, Siegel R, Bandi P and Jemal A: Breast cancer statistics, 2011. CA Cancer J Clin 61: 409-418, 2011.

2. Solomayer EF, Diel IJ, Meyberg GC, Gollan C and Bastert G: Metastatic breast cancer: Clinical course, prognosis and therapy related to the first site of metastasis. Breast Cancer Res Treat 59: 271-278, 2000.

3. Yan Q and Sage EH: SPARC, a matricellular glycoprotein with important biological functions. J Histochem Cytochem 47: 1495-1506, 1999.

4. Bellahcène A and Castronovo V: Increased expression of osteonectin and osteopontin, two bone matrix proteins, in human breast cancer. Am J Pathol 146: 95-100, 1995.

5. Jacob K, Webber M, Benayahu D and Kleinman HK: Osteonectin promotes prostate cancer cell migration and invasion: A possible mechanism for metastasis to bone. Cancer Res 59: 4453-4457, 1999.

6. De S, Chen J, Narizhneva NV, Heston W, Brainard J, Sage EH and Byzova TV: Molecular pathway for cancer metastasis to bone. J Biol Chem 278: 39044-39050, 2003.

7. Helleman J, Jansen MP, Ruigrok-Ritstier K, van Staveren IL, Look MP, Meijer-van Gelder ME, Sieuwerts AM, Klijn JG, Sleijfer S, Foekens JA and Berns EM: Association of an extracellular matrix gene cluster with breast cancer prognosis and endocrine therapy response. Clin Cancer Res 14: 5555-5564, 2008.

8. Hsiao YH, Lien HC, Hwa HL, Kuo WH, Chang KJ and Hsieh FJ: SPARC (osteonectin) in breast tumors of different histologic types and its role in the outcome of invasive ductal carcinoma. Breast J 16: 305-308, 2010.

9. Koblinski JE, Kaplan-Singer BR, VanOsdol SJ, Wu M, Engbring JA, Wang S, Goldsmith CM, Piper JT, Vostal JG, Harms JF, et al: Endogenous osteonectin/SPARC/BM-40 expression inhibits MDA-MB-231 breast cancer cell metastasis. Cancer Res 65: 7370-7377, 2005.

10. Ni X, Xia T, Zhao Y, Zhou W, Wu N, Liu X, Ding Q, Zha X, Sha J and Wang S: Downregulation of miR-106b induced breast cancer cell invasion and motility in association with overexpression of matrix metalloproteinase 2. Cancer Sci 105: 18-25, 2014.

11. van Nijnatten TJA, Moossdorff M, de Munck L, Goorts B, Vane MLG, Keymeulen KBMI, Beets-Tan RGH, Lobbes MBI and Smidt ML: TNM classification and the need for revision of pN3a breast cancer. Eur J Cancer 79: 23-30, 2017.

12. Watkins G, Douglas-Jones A, Bryce R, Mansel RE and Jiang WG: Increased levels of SPARC (osteonectin) in human breast cancer tissues and its association with clinical outcomes. Prostaglandins Leukot Essent Fatty Acids 72: 267-272, 2005.

13. Livak KJ and Schmittgen TD: Analysis of relative gene expression data using real-time quantitative PCR and the 2(-Delta Delta C(T)) method. Methods 25: 402-408, 2001.

14. Marshall MS: Ras target proteins in eukaryotic cells. FASEB J 9: 1311-1318, 1995.

15. Funk SE and Sage EH: The Ca2(+)-binding glycoprotein SPARC modulates cell cycle progression in bovine aortic endothelial cells. Proc Natl Acad Sci USA 88: 2648-2652, 1991.

16. Infante JR, Matsubayashi H, Sato N, Tonascia J, Klein AP, Riall TA, Yeo C, Iacobuzio-Donahue C and Goggins M: Peritumoral fibroblast SPARC expression and patient outcome with resectable pancreatic adenocarcinoma. J Clin Oncol 25: 319-325, 2007.

17. Yamanaka M, Kanda K, Li NC, Fukumori T, Oka N, Kanayama HO and Kagawa S: Analysis of the gene expression of SPARC and its prognostic value for bladder cancer. J Urol 166: 2495-2499, 2001.

18. Iacobuzio-Donahue CA, Argani P, Hempen PM, Jones J and Kern SE: The desmoplastic response to infiltrating breast carcinoma: Gene expression at the site of primary invasion and implications for comparisons between tumor types. Cancer Res 62: 5351-5357, 2002. 
19. Koukourakis MI, Giatromanolaki A, Brekken RA, Sivridis E, Gatter KC, Harris AL and Sage EH: Enhanced expression of SPARC/osteonectin in the tumor-associated stroma of non-small cell lung cancer is correlated with markers of hypoxia/acidity and with poor prognosis of patients. Cancer Res 63: 5376-5380, 2003

20. Sage H, Johnson C and Bornstein P: Characterization of a novel serum albumin-binding glycoprotein secreted by endothelial cells in culture. J Biol Chem 259: 3993-4007, 1984.

21. Prada F, Benedetti LG, Bravo AI, Alvarez MJ, Carbone C and Podhajcer OL: SPARC endogenous level, rather than fibroblast-produced SPARC or stroma reorganization induced by SPARC, is responsible for melanoma cell growth. J Invest Dermatol 127: 2618-2628, 2007.
22. Charest A, Pépin A, Shetty R, Côté C, Voisine P, Dagenais F, Pibarot $\mathrm{P}$ and Mathieu P: Distribution of SPARC during neovascularisation of degenerative aortic stenosis. Heart 92: 1844-1849, 2006.

23. Lien HC, Hsiao YH, Lin YS, Yao YT, Juan HF, Kuo WH, Hung MC, Chang KJ and Hsieh FJ: Molecular signatures of metaplastic carcinoma of the breast by large-scale transcriptional profiling: Identification of genes potentially related to epithelial-mesenchymal transition. Oncogene 26: 7859-7871, 2007. 